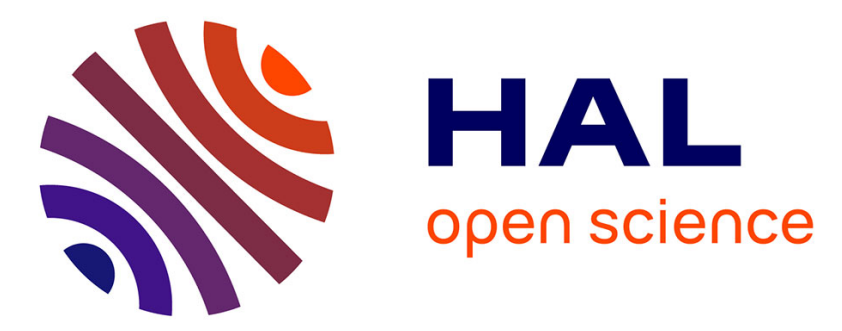

\title{
Application of the theory of Markov chains to model heat and mass transfer between stochastically moving particulate and gas flows
}

\author{
V. Mizonov, Henri Berthiaux, Patricia Arlabosse, D. Djerroud
}

\section{- To cite this version:}

V. Mizonov, Henri Berthiaux, Patricia Arlabosse, D. Djerroud. Application of the theory of Markov chains to model heat and mass transfer between stochastically moving particulate and gas flows. Granular Matter, 2008, 10 (4), pp.335-340. 10.1007/s10035-008-0094-2 . hal-01643400

\author{
HAL Id: hal-01643400 \\ https://hal.science/hal-01643400
}

Submitted on 29 Nov 2017

HAL is a multi-disciplinary open access archive for the deposit and dissemination of scientific research documents, whether they are published or not. The documents may come from teaching and research institutions in France or abroad, or from public or private research centers.
L'archive ouverte pluridisciplinaire HAL, est destinée au dépôt et à la diffusion de documents scientifiques de niveau recherche, publiés ou non, émanant des établissements d'enseignement et de recherche français ou étrangers, des laboratoires publics ou privés. 


\title{
Application of the theory of Markov chains to model heat and mass transfer between stochastically moving particulate and gas flows
}

\author{
V. Mizonov • H. Berthiaux - P. Arlabosse • \\ D. Djerroud
}

\begin{abstract}
A cell model to describe heat and mass transfer between two media, one or both of which are involved in stochastic motion, is proposed. The model consists of two chains of cells to describe 1D stochastic motion of the flows. Each couple of corresponding cells experiences heat and mass exchange at every transition. The model allows describing the transient process as well as the steady-state distribution of parameters of the flows. It allows taking into account such non-linear phenomena as phase transformation (boiling, for example) and the internal heat sources caused, for example, by chemical reactions. The examples of modelling given in the paper demonstrate the ability of the model to describe all necessary features of the process.
\end{abstract}

Keywords Markov chain - Matrix of transition probabilities - Heat and mass transfer - Phase transformation . Stochastic granular flow $\cdot$ Chemical reaction

\section{Introduction}

The thermal treatment of granular materials is widely used in chemical industry. It allows running different physical and chemical processes such as drying, baking, calcining, and others. The classical approach to model and calculate the

V. Mizonov (凶)

Department of Applied Mathematics, Ivanovo Power Engineering State University,

Rabfakovskaya 34, 153003 Ivanovo, Russia

e-mail: mizonov@home.ivanovo.ru

H. Berthiaux · P. Arlabosse - D. Djerroud

Laboratoire de Génie des Procédés des Solides Divisés,

UMR CNRS 2392, Ecole des Mines d'Albi - Carmaux, 81000 Albi, France

e-mail: berthiau@enstimac.fr process is based on the integral heat and mass balance equations written for input and output parameters of the process $[1,2]$. It does not take into account local distributions of the parameters over an apparatus, i.e., local conditions of heat and mass transfer and heat and mass emission, which can vary strongly over the apparatus. Usually this approach is accompanied by a large number of correcting coefficients taken from experiments at an already functioning apparatus.

The description of the processes on the basis of the local approach employs differential equations of flows motion and heat and mass transfer between them $[3,4]$. Analytical solutions to the equations are possible only under considerable simplifications and require special knowledge in mathematics that makes them not very convenient for engineers. These solutions are very useful for understanding of the process behavior but they can hardly be used as a realistic basis for their calculation, particularly if the process includes obviously non-linear phenomena such as phase transformations or chemical reactions.

The cell models, mostly based on the theory of Markov chains, occupy the intermediate position between these two approaches. They operate with the local distributions where the scale of locality is not infinitely small but coincides with a chosen cell size. This size can be conditioned by the design of an apparatus, for example, by sections covered by transporting or mixing blades.

Markov chains have been employed to model particulate flows in a great variety of process equipments. Inoue and Yamaguchi [5] for a V- blender, Chen et al. [6] and then Lai and Fan [7] for static mixers, Boss and Dabrowska [8] for a silo mixer, Fox and Fan [9] and more recently Dehling et al. [10], or Harris et al. [11] are good examples of Markov chain modeling of bulk particulate flows. Mixing of different flows have also been investigated by either homogeneous or heterogeneous Markov chains, as can be seen in papers 
by Oyama and Ayaki [12], Fan and Shin [13] or Dechsiri et al. [14].

The strategy of application of the approach to describe the stochastic motion of a single-phase flow was discussed in [15-18]. At the present study an attempt to use the approach to describe the process, in which two components move stochastically and experience heat and mass exchange, is undertaken.

\section{Basic chain model and governing equations}

\subsection{Structure of the chain model}

Let us suppose that there are two parallel flows (for example, the particulate flow 1 and the gas flow 2), which can exchange with heat and/or mass (Fig. 1). Let us divide the total length of the process into the finite number $m$ of perfectly mixed cells of identical length $\Delta \mathrm{x}$. The choice of $\mathrm{m}$ can be conditioned by different reasons and should be discussed in each particular case. For example, if blades transport the particulate flow, the value of $m$ can be taken as the number of zones covered by the blades. If we want to obtain the process description, which is more close to the continuous dispersion equation, the value of $\mathrm{m}$ (and related value of $\Delta \mathrm{x}$ ) should be chosen from the condition when the obtained distributions of flow parameters stop depending on $\Delta \mathrm{x}$ with a reasonable accuracy. It is necessary to note that the choice of $m$ ifluences on transition probabilities that will be discussed below.

If we examine evolution of the additive property $\mathrm{S}$ of a flow, its distribution over the process length can be presented as the state column vector $\mathbf{S}=\left[\mathrm{S}_{1}, \mathrm{~S}_{2}, \ldots \mathrm{S}_{m}\right]^{\mathrm{T}}$ where the upper symbol ${ }^{\mathrm{T}}$ means transposing of a vector, or matrix. Also suppose that we observe the state of the process at discrete moments of time $t_{i}=i \Delta t$ where $\Delta t$ is the transition duration, and $i$ is the number of transition, which can be interpreted as discrete time. During $\Delta t$ the state $S_{\mathrm{j}}$ in the $\mathrm{j}$-th cell can be changed due to the following reasons: the action of external sources, which can be subdivided into the feed to the chain $\Delta S_{\mathrm{jf}}$, and the amount of $\mathrm{S}$ that comes form the corresponding cell of the neighboring chain $\Delta S_{\mathrm{j} 1-2}$; the action of internal

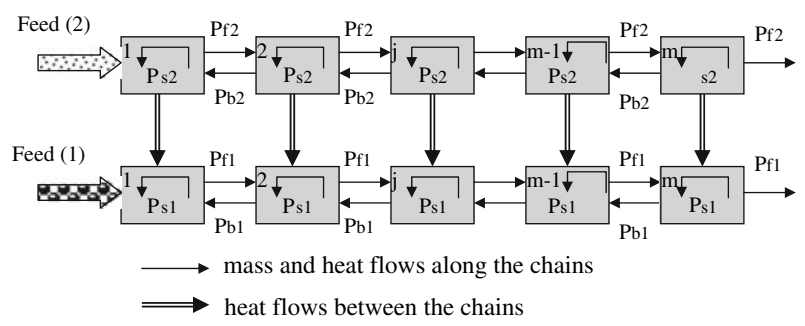

Fig. 1 The chain model of the process with heat exchange between chains sources $\Delta S_{\mathrm{j} \text { int }}$ caused, for example, by chemical reactions; the longitudinal transition of the property along the chain due to the stochastic motion of the medium the property $\mathrm{S}$ belongs to. As far as the changes are similar for all cells of the chain, they also can be presented in the vector (matrix) notation, and the kinetic equation of the evolution of the property can be written in the following form:

$\mathbf{S}^{\mathbf{i}+\mathbf{1}}=\mathbf{P}\left(\mathbf{S}^{\mathbf{i}}+\Delta \mathbf{S}_{\mathbf{f}}^{\mathbf{i}}+\Delta \mathbf{S}_{1-2}^{\mathbf{i}}+\Delta \mathbf{S}_{\mathrm{int}}^{\mathrm{i}}\right)$

where $\mathbf{P}$ is the matrix of transition probabilities, which describes the stochastic motion of the medium along the chain and will be discussed below.

\subsection{Matrices of transition probabilities}

The transition duration should be small enough the particles in a cell could be able to transit only to the neighboring cells. Thus, during $\Delta \mathrm{t}$ a particle can stay inside the cell, or get the forward or backward transition. The probabilities of these transitions are $P_{s}, P_{f}$ and $P_{b}$ respectively. It is obvious that for any cell, which is not open outside the chain, $P_{s}+P_{f}+$ $P_{b}=1$. The transition probabilities can be arranged as the tridiagonal matrix where the probabilities to stay are placed on the main diagonal of it, and the forward and backward probabilities are placed below and above this diagonal.

Two approaches can be used to define the transition probabilities. The first one is based on direct experimental study of the flow motion with the help of its tracing and using the RTD-technique. The details of the approach with application to modeling of continuous blade mixer by means of the theory of Markov chains can be found in our paper [18].

The second approach is based on similarity of the chain model and the finite difference procedure for numerical solution of the dispersion equation, which is also often used to model particulate flows. The matrix of transition probabilities construction on the basis of this approach is described below.

In many practical cases it is convenient to subdivide the transitions into completely random (symmetrical) transitions and deterministic transition. If a flow moves to the right (Fig. 1) this operation gives for all intermediate cells: $P_{b}=d$, $P_{f}=d+v, P_{s}=1-2 d-v$ where d is related to completely stochastic motion $\left(d=D \Delta t / \Delta x^{2}\right.$ where $D$ is the dispersion coefficient, which remains the adjusting parameters to agree the experimental and calculated RTD curve in most of models), and $v$ is related to its deterministic component $(v=V \Delta t / \Delta \mathrm{x}$ where $V$ is the mean velocity of medium motion, which is to be calculated from the continuity equation if the hold-up and throughput are known). In this notation the matrix of transition probabilities has the form: 
$\mathbf{P}=\left[\begin{array}{ccccccc}1-d-v & d & 0 & \ldots & 0 & 0 & 0 \\ d+v & 1-2 d-v & d & \ldots & 0 & 0 & 0 \\ 0 & d+v & 1-2 d-v & \ldots & 0 & 0 & 0 \\ \ldots & \ldots & \ldots & \ldots & \ldots & \ldots & \ldots \\ 0 & 0 & 0 & \ldots & 1-2 d-v & d & 0 \\ 0 & 0 & 0 & \ldots & d+v & 1-2 d-v & d \\ 0 & 0 & 0 & \ldots & 0 & d+v & 1-d-v\end{array}\right]$

It is necessary to note that the matrix in Eq. (2) is built for the cells of identical volume. Otherwise the correction coefficients should appear at the transition probabilities. Despite it is written for $v=$ const and $d=$ const, no problems arise if to take into account the variation of these parameters along the process if corresponding correlations between them and flow parameters are known. At the constant feed to the first cell and absence of all source terms in Eq. (1) the matrix given by Eq. (2) provides the homogeneous asymptotic distribution of $S$ over the cells.

The problem of reliability of determination of the transition probabilities arises here. It can be checked by the possibility to correspond the experimental and calculated RTD curves using only one adjusting parameter $D$. If the correspondence cannot be reached, it means that the onedimensional chain of perfectly mixed cells does not fit the real flow structure, and it is necessary to employ a multidimensional array of the cells describing the nonhomogeneous flow in the crosswise direction. This is also can be done on the basis of the theory of Markov chains but such complication of the model is beyond the present study.

\subsection{Flows between chains and internal sources}

First let us examine the case of direct-current exchange. Let the chain for granular material flow is being fed through the left cell as it is shown in Fig. 1. The total feed rate $G_{10}$ consists of the feed rate of dry matter $D_{10}$ and the feed rate of moisture $W_{10}$ that means that the dry basis moisture content is $X_{10}=W_{10} / D_{10}$. The temperature of the material $T_{10}$ is also known.

The feed vectors for the chain are:

dry component in the flow 1

$\Delta \mathbf{D}_{\mathbf{1 f}}=\left[D_{10} \Delta t, 0,0, \ldots, 0\right]^{T}$

moisture in the flow 1

$\Delta \mathbf{W}_{\mathbf{1 f}}=\left[W_{10} \Delta t, 0,0, \ldots, 0\right]^{T}$

heat with the flow 1

$\Delta \mathbf{Q}_{1 \mathbf{f}}=\left[D_{1} c_{1 d} T_{10} \Delta t+W_{1} c_{1 w} T_{10} \Delta t, 0,0, \ldots, 0\right]^{T}$

where $c_{1 d}$ and $c_{1 w}$ are the specific heats of the components of the flow 1 .
It is supposed that all these quantities are transported along the chain 1 by the matrix $\mathbf{P}_{\mathbf{1}}$, which is given by Eq.(2) with $\mathrm{v}_{1}$ and $\mathrm{d}_{1}$.

The same formulae can be used to construct the feed vectors for the flow 2, motion of which along the chain is controlled by the matrix $\mathbf{P}_{2}$. In order to transit form the directcurrent exchange to the coutercurrent one it is just necessary to rotate $180^{\circ}$ the matrix $\mathbf{P}_{2}$ and the feed vectors for the flow 2.

The amount of heat transiting from a cell of the chain 2 to the corresponding cell of the chain 1 can be described as follows (indices are omitted):

$\Delta Q_{2}=\alpha_{t} F\left(T_{2}-T_{1}\right) \Delta t=\mathrm{a}_{t}\left(T_{2}-T_{1}\right)$

where $\alpha_{t}$ is the heat transfer coefficient from gas to granular material, $\mathrm{F}$ is the surface of heat exchange in the cell.

The process of heat transfer between couples of cells of parallel chains (Eq.(6)) is illustrated in Fig. 2 where the process for one-component flows without mass transfer is shown. Being a non-additive property, the temperature cannot travel between and along the chains but its difference defines the heat traveling over the cells. The same can be said about the moisture content. This is why all the transitions are defined for heat and mass but temperatures and moisture contents are to be calculated after each transition to calculate heat and mass transitions during the next time step.

This heat goes to heating the cell of the flow $1\left(\Delta Q_{1}\right)$ and/or to evaporation or vaporization of moisture from it:

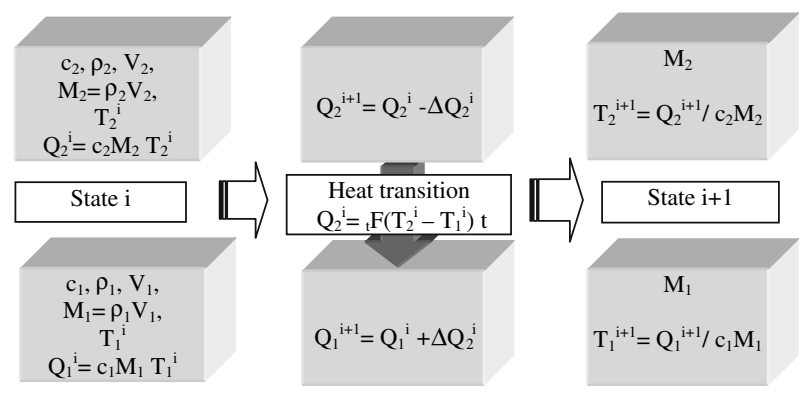

Fig. 2 Heat transition between the couple of cells of parallel chains and new thermal states of the cells 


$$
\begin{aligned}
\Delta Q_{1} & =\Delta Q_{2}-q_{e v} \Delta W_{1}, \Delta W_{1}=\alpha_{u} F\left(U_{2}-U_{1}\right) \Delta t \\
& =a_{u}\left(U_{2}-U_{1}\right) \quad \text { if } T_{1}<T_{b}, W_{1}>0 \\
\Delta Q_{1} & =0, \Delta W_{1}=\Delta Q_{2} / \mathrm{q}_{v} \quad \text { if } T_{1}=T_{b}, W_{1}>0
\end{aligned}
$$

where $\alpha_{u}$ is the mass (moisture) transfer coefficient from granular material to gas, $q_{e v}$ and $q_{v}$ is the specific heat of evaporation and vaporization respectively, $U$ is the moisture concentration. If moisture is completely removed from the flow $1\left(W_{1}=0\right), \Delta Q_{2}$ goes to further heating of dry matter of the flow 1 .

After the heats and the masses are removed along the chains by the transition matrices (Eq.1) and the next states are calculated, the next vectors of temperature can be found as

$T_{1,2}^{i+1}=Q_{1,2}^{i+1} \cdot /\left(c_{d 1,2}^{i+1} D_{1,2}^{i+1}-c_{w 1,2}^{i+1} W_{1,2}^{i+1}\right)$

where the operator ./ means element by element (scalar) division of vectors. The similar expression can be written for the moisture concentration.

In order to introduce the internal source caused by a chemical reaction let us suppose that after the temperature $T_{1}$ reaches the value $T_{r o}$ the reaction of thermal decomposition of the component A with the specific heat of reaction $q_{r}$ begins in the granular flow $\left(q_{r}>0\right.$ for an exothermic reaction and $q_{r}<0$ for an endothermic one). Let it be a reaction of the first order, the rate of which is subjected to the Arrhenius low:

$$
\begin{aligned}
& \frac{d c_{A}}{d t}=-k_{A} c_{A} \quad \text { with } k_{A}=0 \text { at } T_{1}<T_{r o} \text { and } \\
& k_{A}=z_{A} e^{-\frac{E_{A}}{R\left(T_{1}-T_{r 0}\right)}}=z_{A} e^{-\frac{E_{A}}{R\left(T_{20}-T_{0}\right)} \frac{\left(T_{20}-T_{r 0}\right)}{\left(T_{1}-T_{r 0}\right)}} \\
& =z_{A} \beta^{\frac{\left(T_{20}-T_{0}\right)}{\left(T_{1}-T_{0}\right)}} \text { at } T_{1}>T_{\text {ro }}
\end{aligned}
$$

The variation of concentration of $A$ in the dry component $D_{1}$ of the flow 1 during $\Delta t$ will cause the following heat in the cell:

$$
\begin{aligned}
\Delta Q_{r}=q_{r} \Delta D_{1 A} & =q_{r} D_{1 A} z_{A} \beta^{\frac{\left(T_{20}-T_{r 0}\right)}{\left(T_{1}-T_{r 0}\right)}-1} \Delta t \\
& =q_{r r} D_{1 A} \beta^{\frac{\left(T_{20}-T_{r 0}\right)}{\left(T_{1}-T_{r 0}\right)}-1}
\end{aligned}
$$

where $q_{r r}=q_{r} z_{A} \Delta t$ is the reduced heat of the reaction.

\section{Some results of modelling}

These results are shown in Figs. 3-5 where asymptotic distributions of parameters over the cells are presented. It is necessary to note that the objective of the section is not to model this or that real process but to demonstrate workability of the proposed mathematical tool in wide range of sub-processes involved. This is why the examples may look artificial but they are constructed to emphasize the influence of this or that process parameter. All temperatures on the graphs below are normalized to the hot gas temperature and are dimensionless. The following can be said about the dimensionless transfer parameters $a_{t}$ and $a_{u}$. They include not only the physical process parameters but also the parameters of modeling $\Delta t$ and $\Delta x$. The value of these parameters $a_{t}$ and $a_{u}$ must be within the range 0 (no exchange) and 1 . If one of them is higher than 1 , the computational stability is being lost. Physically it mean that during a transition the value of a property extracted from a cell is bigger than the cell contains. The situation can be corrected by reduction of $\Delta t$. The similar requirement comes from the transition matrix side, in which the elements on the main diagonal should not be negative. Thus, the transition duration must be less than the smallest one providing the computational stability. In the examples below the values of the transfer coefficient are taken more or less arbitrary from the range providing the computational stability. They have no qualitative influence on the distributions of flows parameters, and are important only in
Fig. 3 Influence of the solid flow axial dispersion (left) and the flow rate of solids (right) on temperature and moisture content distribution for the direct-current exchange: $a_{t}=0.03, a_{u}=0.01, X_{10}=$ $0.1, q_{v}=10$ (black markers material flows, white markers gas flows)
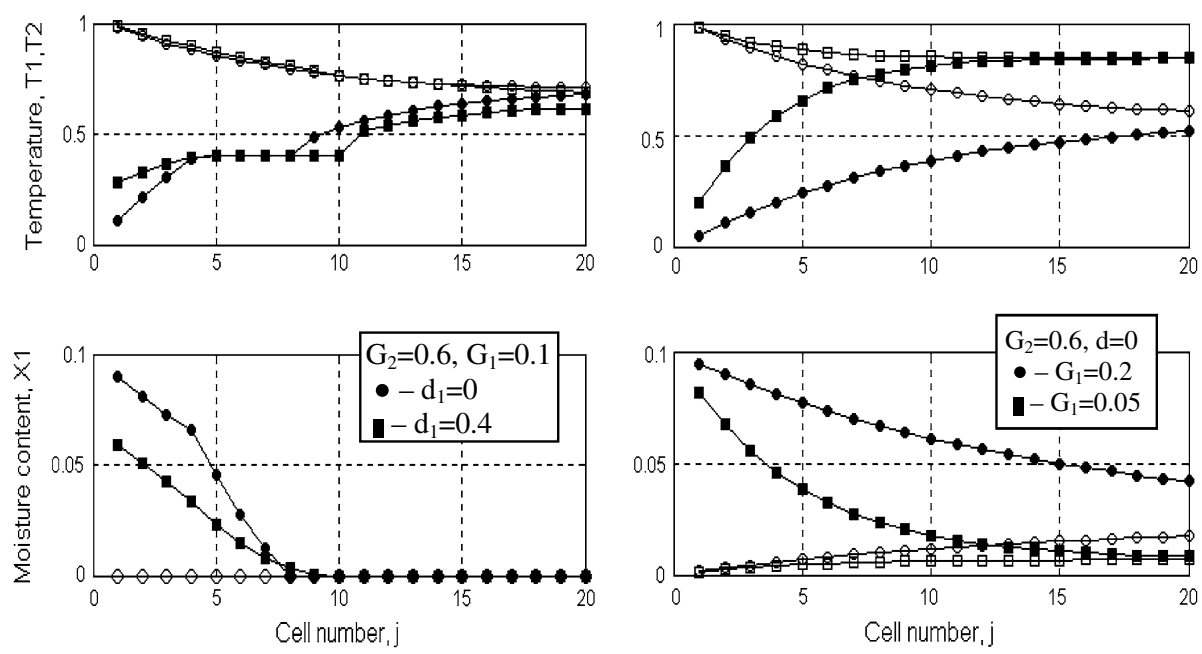
Fig. 4 Influence of the flow rate of solids (left) and the heat of vaporization (right) on temperature and moisture content distribution for the countercurrent exchange: $a_{t}=$ $0.06, a_{w}=0.01, X_{10}=0.1$
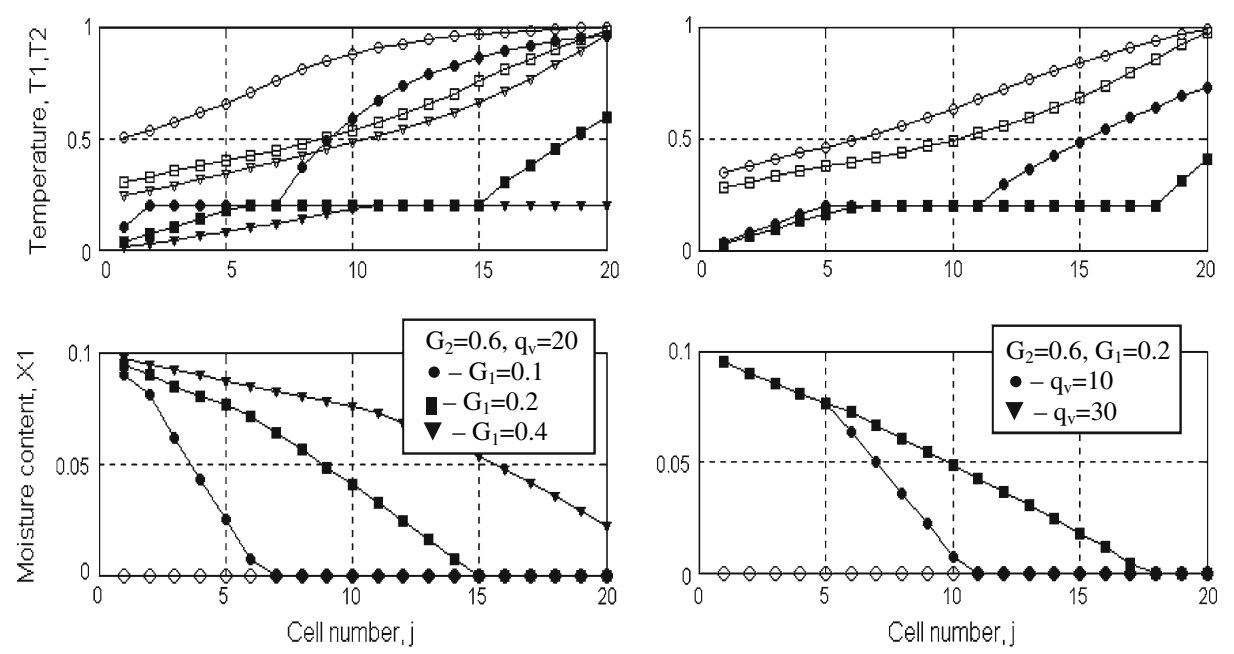
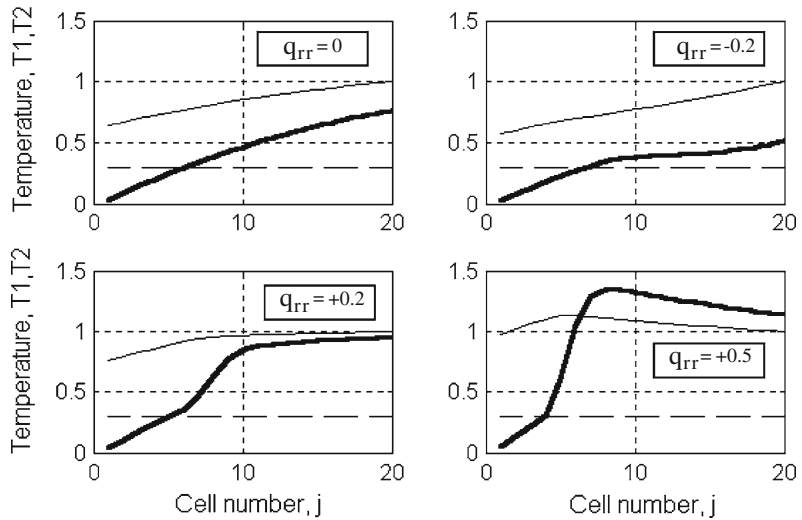

Fig. 5 Influence of the reduced heat of chemical reaction on temperature distribution for the countercurrent heat exchange: $a_{t}=0.05, z_{a}=$ $0.5, T_{r 0}=0.3$. (thin lines gas temperature, solid lines material temperature, dotted lines temperature threshold of chemical reaction)

the case of modeling of a particular process, which is not the objective of the present study.

Figure 3 shows the influence of the solid flow axial dispersion (left) and the flow rate of solids (right) on the temperature and moisture content distribution for the direct-current exchange. The left graphs are related to the unlimited moisture absorption capacity by the flow 2 and boiling temperature equal to $0.4 T_{20}$. The stochasticity of the granular flow affects strongly on distribution of parameters of this flow. Boiling begins closer to the wet granular flow inlet and ends more far from it for higher level of axial stochasticity of the granular flow. However, the dry remainder of the flow 1 at the outlet has less temperature for this case. Evacuation of water from the flow 1 (drying) goes faster at the beginning but the very last portions of water are kept longer in the flow with higher stochasticity. The graphs on the right demonstrate how the ratio of flow rates affects on the distributions (boiling is not taken into account in this example). The physical sense of the influence is obvious.

Figure 4 shows the influence of the flow rate of solids (left) and the heat of vaporization (right) on temperature and moisture content distribution for the countercurrent exchange.

At small flow rate of the wet granular material (circles) boiling goes very close to its inlet and occupy rather short distance of the process. The dry remainder of the flow at its outlet has the temperature, which is close to gas temperature at its inlet. At higher flow rate of the wet granular material (squares) boiling begins later, occupies longer zone of the process but ends before the outlet is reached. The dry remainder is heated up but to lower temperature than in the previous case. At last, for the case of more high flow rate (triangles) boiling begins in the middle of the process and does not end at the outlet. The flow still contains some water and has the temperature equal to the temperature of boiling.

As far as the wet component of the flow 1 is not necessarily water, the graphs on the right show the influence of the heat of vaporization on the process parameters distribution. The physical sense of the influence is also obvious. At higher heat of vaporization the zone of boiling is longer, and due to higher temperature drop of the flow 2 boiling begins farther from the granular flow inlet.

Influence of the reduced heat of chemical reaction on temperature distribution for the countercurrent heat exchange is shown in Fig. 5.

The upper left graph is the basis for comparison. The endothermic reaction leads to a quasi-isothermal zone on the heating curve of the flow 1 ; the exothermic reaction leads to the sharp growth of the curve. If the specific heat of the reaction is high enough (in combustion, for example), the temperature of the flow 1 can exceed the temperature of the flow 2, and the flow 1 becomes the heating medium for the flow 2. 


\section{Conclusions}

The Markov chain model to describe heat and mass transfer between stochastically moving particulate and gas flows is proposed. The model is based on the universal algorithm of axial transitions of mass, heat and moisture from one cell to another during a transition, and on presenting of mass, heat and moisture coming from, or going to the neighbouring flow as the source terms within the transition. The model allows taking into account such non-linear phenomena as phase transformation (boiling, for example) and the internal heat sources caused, for example, by chemical reactions.

Some results of modeling are presented to demonstrate workability of the proposed tool. In particular, it is shown computationally that the growth of granular flow axial stochasticity extends the zone of boiling and, respectively, the zone of water evacuation despite the moisture content near the inlet decreses faster. It is also shown that if an exothemic chemical reaction occurs in the granular flow and the specific heat of the reaction is high enough, there can be inversion of heating and heated medium when the granular flow becomes the heating medium for already hot gas. The quantitative influence of some other process parameters on the steadystate distributions of temperature and moisture content are also presented.

The obvious advantage of the approach is that the only mathematical tool, which is necessary to construct and run the model, is the basics of matrix algebra. The examples of modelling given in the paper demonstrate the ability of the model to describe all necessary features of the process.

\section{References}

1. Stoecker, W.F.: Design of thermal systems. McGraw-Hill Book Co., NY (1989)

2. Strumillo, C., Kudra, T.: Drying: principles, applications and design. Gordon \& Breach Science Publishers, Montreux (1986)
3. Oran, E.S., Boris, J.P.: Numerical simulation of reactive flow. Elsevier, Amsterdam (1987)

4. Oakley, D.E., Bahu, R.E.: Computational modeling of spray dryers. Computers \& Chemical Engineering 17, 493-498 (1993)

5. Inoue, I., Yamaguchi, K.: Particle motion in a mixer. Mixing in a two dimensional V-type mixer. Int Chem Eng 10(3), 490-497 (1970)

6. Chen, S.J., Fan, L.T., Watson, C.A.: The Mixing of solid particles in a motionless mixer-a stochastic approach. AIChE J 18(5), 984-989 (1972)

7. Lai, F.S., Fan, L.T.: Application of a discrete mixing model to the study of mixing of multicomponent solid particles. Ind Eng Chem Process Des Dev 14(4), 403-411 (1975)

8. Boss, J., Dabrowska, D.: Stochastic model of mixing during discharging of granular materials from a bin-I: two-component system. J Powder Bulk Solids Tech 9(4), 1-11 (1985)

9. Fox, R.O., Fan, L.T.: Stochastic analysis of axial solids mixing in a fluidised bed. In: Proceedings of the 1st World Congress on Particle Technology Part III, pp 581-595. Nurnberg (1986)

10. Dehling, H.G., Hoffman, A.C., Stuut, H.W.: Stochastic models for transport in a fluidised bed. SIAM J Appl Math 60(1), 337358 (1999)

11. Harris, A.T., Thorpe, R.B., Davidson, J.F.: Stochastic modelling of the particle residence time distribution in circulating fluidised bed risers. Chem Eng Sci 57, 4779-4796 (2002)

12. Oyama, Y., Agaki, K.: Studies on the mixing of particulate solids. Kagaku Kogaku (Japan) 20, 148-154 (1956)

13. Fan, L.T., Shin, S.H.: Stochastic diffusion model of non-ideal mixing in a horizontal drum mixer. Chem Eng Sci 34, 811-820 (1979)

14. Dechsiri, C., Abanades, J.C., Dehling, H.G., Hoffman, A.C., Wright, P.C.: A stochastic model for mixing an segregation in slugging fluidised beds. Récents Progrès En Génie Des Procédés 14(76), 631-638 (2000)

15. Tamir, A.: Applications of Markov chains in chemical engineering. Elsevier, Amsterdam (1998)

16. Berthiaux, H., Mizonov, V.: Applications of Markov chains in particulate process engineering: a review. Can J Chem Eng 85(6), 1143-1168 (2004)

17. Berthiaux, H., Mizonov, V., Zhukov, V.: Application of the theory of Markov chains to model different processes in particle technology. Powder Tech 157, 128-137 (2005)

18. Marikh, K., Berthiaux, H., Mizonov, V. et al.: Flow analysis and Markov chain modeling to quantify the agitation effect in a continuous mixer. Chem Eng Res Des 84(A11), 1059-1074 (2006) 